\title{
Bounding quantum-gravity-inspired decoherence using atom interferometry
}

\author{
Jiří Minár., ${ }^{1}$ Pavel Sekatski, ${ }^{2}$ and Nicolas Sangouard ${ }^{3}$ \\ ${ }^{1}$ School of Physics and Astronomy, University of Nottingham, Nottingham NG7 2RD, United Kingdom \\ ${ }^{2}$ Institut für Theoretische Physik, Universität Innsbruck, Technikerstraße 21a, A-6020 Innsbruck, Austria \\ ${ }^{3}$ Department of Physics, University of Basel, Klingelbergstrasse 82, 4056 Basel, Switzerland
}

(Received 25 October 2016; published 12 December 2016)

\begin{abstract}
Hypothetical models have been proposed in which explicit collapse mechanisms prevent the superposition principle from holding at large scales. In particular, the model introduced by Ellis et al. [J. Ellis et al., Phys. Lett. B 221, 113 (1989)] suggests that quantum gravity might be responsible for the collapse of the wave function of massive objects in spatial superpositions. We consider here a recent experiment reporting on interferometry with atoms delocalized over half a meter for a time scale of $1 \mathrm{~s}$ [T. Kovachy et al., Nature (London) 528, 530 (2015)] and show that the corresponding data strongly bound quantum-gravity-induced decoherence and rule it out in the parameter regime considered originally.
\end{abstract}

DOI: 10.1103/PhysRevA.94.062111

\section{INTRODUCTION}

While the structure of space-time at the Planck scale is not known in detail, one expects that it is a subject to quantum fluctuations. It has been suggested that the departure from flat space-time on short distances can degrade the spatial coherences of massive systems. In particular, Ellis et al. have proposed that nontrivial configurations of spacetime-wormholes-might lead to the decoherence of spatial superpositions of massive objects (see the model presented in Ref. [1] and further elaborated in Ref. [2]). The basic idea is that a massive object initially prepared in a superposition of two different locations entangles with degrees of freedom of the wormholes, which would leave the object in a classical mixture once the wormholes are traced out. It provides an explicit mechanism for a quantum-classical crossover. It is also an example of a phenomenological approach in which falsifiable predictions might provide hints about quantum gravity [see, e.g., [3,4] for string-theory-inspired models (or [5] for a review of various phenomenological models)].

We note that while Coleman suggested the wormhole theory as a possible solution to the cosmological constant problem [6], it was argued soon after that the theory would lead to a wormhole-dominated universe [7-10]. Despite such an argument (see also [11,12] for other arguments and [13] for historical overview and discussion), the model of Ellis et al. has been considered in many proposals for testing quantum-gravity-induced decoherence including particle decay experiments [14,15], superconducting devices [16,17], atomic and photon interferometry [18-23], cavity quantum optomechanics with nano- and micromechanical oscillators [24-32], or using dielectric nanospheres [33-35]. While challenging experiments are being set up to implement these proposals, some of them forcing experimentalists to envision experiments in space [36], we have pointed out in [37] that simple techniques with single atoms trapped in optical lattices could be used to test efficiently the model of Ref. [2]. In particular, we have shown that quantum-gravity-induced decoherence can have a significant effect on the state of a single atom if the latter is delocalized on centimeter scales for a time of the order of a few seconds.
With this in mind, we welcome the experimental results presented recently in Ref. [38], where a quantum superposition of rubidium atoms at the half-meter scale was achieved using light-pulse interferometry. Here we show that the corresponding data set strong bounds on the model of Ref. [2] and rule it out in the parameter regime that was considered originally.

\section{PRINCIPLE}

In this section we summarize the principle of quantumgravity-induced decoherence, arising from a phenomenological treatment $[1,2]$ of the interaction of matter with a dilute-gas wormhole background. We consider a system with mass $m$, described initially by the state $\rho_{0}$. In the model of Ref. [2], an unperturbed wormhole is described by a state $\rho_{w}=\left|\psi_{w}\right\rangle\left\langle\psi_{w}\right|$, which is assumed to be pure and have a Gaussian wave function in the momentum space $\left|\psi_{w}\right\rangle=\int d^{3} \mathbf{p} \psi(\mathbf{p})|\mathbf{p}\rangle$, where $\psi(\mathbf{p}) \propto e^{-p^{2} /(2 \sigma)^{2}}$. It has zero mean momentum and a spread $\sigma \sim \mathrm{cm}_{0}^{2} / \hbar m_{\mathrm{Pl}} \sim 10^{-3} \mathrm{~m}^{-1}$, where $m_{0}$ is the mass of the nucleon, $m_{\mathrm{Pl}}$ the Planck mass, $c$ the speed of light, and $\hbar$ the reduced Planck constant. The interaction between the wormhole and the system is treated in the input-output $S$-matrix formalism and is assumed to result in the elastic scattering of the wormholes on the system. Concretely, at any time there is a small probability amplitude that a wormhole scatters according to $|\mathbf{p}\rangle \rightarrow$ $i \int d^{3} \mathbf{p}^{\prime} e^{i\left(\mathbf{p}^{\prime}-\mathbf{p}\right) \cdot \mathbf{X}} \delta\left(|\mathbf{p}|-\left|\mathbf{p}^{\prime}\right|\right) \frac{F\left(\mathbf{p}^{\prime}\right)}{\left|\mathbf{p}^{\prime}\right|}\left|\mathbf{p}^{\prime}\right\rangle$, which entangles it with system's position given by the operator $\mathbf{X}$. Here $F(\mathbf{p})$ are the dimensionless scattering amplitudes. Accordingly, once the wormhole is traced out, the effect of such a scattering event on the system is a decay of coherence terms $\left|\mathbf{x}^{\prime}\right\rangle\langle\mathbf{x}| \rightarrow$ $r\left|\mathbf{x}^{\prime}\right\rangle\langle\mathbf{x}|$ by some factor $r$ that depends on the spatial spread $\mathbf{x}-\mathbf{x}^{\prime}$. As long as the typical wavelength of wormholes $\frac{1}{\sigma} \gg\left|\mathbf{x}-\mathbf{x}^{\prime}\right|$ dominates the system spatial spread, the decay grows quadratically with the spatial spread $r \propto\left|\mathbf{x}-\mathbf{x}^{\prime}\right|^{2}$. This dependence follows from the Taylor expansion of $e^{i\left(\mathbf{p}^{\prime}-\mathbf{p}\right) \cdot \mathbf{x}}$ [2]. It can be intuitively understood as $r$ is given by the overlap of two scattered modes centered at $\mathbf{x}$ and $\mathbf{x}^{\prime}$, which carry the information about the system position. In fact, in the other extreme $\frac{1}{\sigma} \ll\left|\mathbf{x}-\mathbf{x}^{\prime}\right|$ the localization rate resulting from 
scattering becomes independent of the spread and saturates to a constant value $[39,40]$. While such a possibility might be accounted for in the study of the decoherence mechanism, for the remainder of this work we focus on the former case $\frac{1}{\sigma} \gg\left|\mathbf{x}-\mathbf{x}^{\prime}\right|$ corresponding to the original considerations of Ref. [2] and spatial separations of the order of a meter achievable in today's atomic interferometry.

Since the scattering events have a low probability and happen randomly, the interaction of the system with a dilutegas of wormholes results in the addition of a time-independent localization term to the master equation

$$
\dot{\rho}=i\left[\rho, H_{0}\right]-\frac{1}{2} \int d^{3} \mathbf{x} d^{3} \mathbf{x}^{\prime} \gamma_{\mathrm{QG}}\left|\mathbf{x}-\mathbf{x}^{\prime}\right|^{2}|\mathbf{x}\rangle\left\langle\mathbf{x}|\rho| \mathbf{x}^{\prime}\right\rangle\left\langle\mathbf{x}^{\prime}\right|,
$$

where $H_{0}$ stands for the unitary evolution of the system and it is assumed that the characteristic interaction time of a wormhole with the system is much smaller than the time scales of $H_{0}$. The resulting localization rate coefficient $\gamma_{\mathrm{QG}}$ depends on the wormhole momentum spread $\sigma$; the scattering amplitudes $F(\mathbf{p})$, which are functions of the system mass $m$; and the density of wormholes, which is assumed to be $O(1)$ per Planck volume by Ellis et al. and is given by [2]

$$
\gamma_{\mathrm{QG}}=\frac{\left(c m_{0}\right)^{4} m^{2}}{\left(\hbar m_{\mathrm{Pl}}\right)^{3}}
$$

While the modification (1) is appealing as an attempt to explain the absence of coherence of macroscopic objects, we argue in the next section that the experimental results of Ref. [38] are in contradiction with the model of Ref. [2], at least for the parameter regime presented in Ref. [2].

\section{EXPERIMENT}

Here we briefly describe the experiment reported in Ref. [38]. Kovachy et al. start by launching a Bose-Einstein condensate made with about $10^{5}{ }^{87} \mathrm{Rb}$ atoms in a 10 -m-high atomic fountain. Once launched, a sequence of pulses is applied to control the atom momenta so that the wave packet of each atom is split and recombined coherently to form the analog of a Mach-Zehnder interferometer. In the experiment presented in Ref. [38], the atomic wave packets get separated during a drift time $T=1.04 \mathrm{~s}$, after which they reach their maximum separation $d_{\max }$. They are then recombined to spatially overlap after another drift interval $T=1.04 \mathrm{~s}$. The spatial separation $d_{\max }=n(\hbar k / m) T$ depends on the laser pulse wave number $k$, the number of photon recoils at the first atom splitter $n \hbar k$, and the atomic mass $m$. In Ref. [38], an atom splitter transferring up to $90 \hbar k$ photon recoils is obtained using $2 \hbar k$ Bragg transitions [41], which results in a distance $d_{\max }=54 \mathrm{~cm}$. The contrast of the interference is determined by measuring the variation of the normalized number of atoms in one of the two outputs of the interferometer. For $d_{\max }=54 \mathrm{~cm}$, the measured contrast is of $28 \%$. Here the loss of coherence is in agreement with the measured atom loss in the interferometer (see Fig. 4b in [38]).

\section{BOUNDING QUANTUM-GRAVITY-INDUCED DECOHERENCE}

Let $d(t)$ be the time-dependent function describing the delocalization of a single ${ }^{87} \mathrm{Rb}$ atom. According to Eq. (1), the coherence term of the delocalized atom evolves as $\left\langle\mathbf{x}|\rho(t)| \mathbf{x}^{\prime}\right\rangle=$ $\exp \left[-\gamma_{\mathrm{QG}} \int_{0}^{2 T} d^{2}(t) d t\right]\left\langle\mathbf{x}\left|\rho_{0}\right| \mathbf{x}^{\prime}\right\rangle$. Assuming $d(t)=d_{\max }+$ $\frac{d_{\max }}{T}\left(\frac{T}{2 \pi} \sin \frac{2 \pi|t-T|}{T}-|t-T|\right)$ [42], we have $\left\langle\mathbf{x}|\rho(t)| \mathbf{x}^{\prime}\right\rangle \approx$ $e^{-0.8 \gamma_{\mathrm{QG}} d_{\max }^{2} T}\left\langle\mathbf{x}\left|\rho_{0}\right| \mathbf{x}^{\prime}\right\rangle$ and the contrast of the corresponding interference is

$$
C \approx 2\left\langle\mathbf{x}\left|\rho_{0}\right| \mathbf{x}^{\prime}\right\rangle e^{-0.8 \gamma_{\mathrm{QG}} d_{\max }^{2} T}
$$

It is interesting to analyze the implications of experimental results reported in [38] on the localization rate coefficient $\gamma_{\mathrm{QG}}$. The reduction of the observed contrast is attributed to the atom loss in the interferometer, which is obtained using an independent measurement. The mechanism of Ref. [2] must thus be compatible with the error bars reported in Fig. 4b of [38], which are of the order of $1 \%$. Lets thus assume that the wormholes are responsible for a $1 \%$ decrease in the contrast, $C=0.99$. Replacing $\gamma_{\mathrm{QG}}$ in Eq. (3) by $\gamma_{\mathrm{QG}}^{\prime}$, we find that $\gamma_{\mathrm{QG}}^{\prime}=0.04 \mathrm{~s}^{-1} \mathrm{~m}^{-2}$. This contrasts with the value $\gamma_{\mathrm{QG}}=106 \mathrm{~s}^{-1} \mathrm{~m}^{-2}$ [given by Eq. (2) with $m$ the ${ }^{87} \mathrm{Rb}$ mass]. Equivalently, using $\gamma_{\mathrm{QG}}$ in Eq. (3) yields $C \approx 10^{-11}$. Even if the trajectory of the atoms can be different from the one that we have considered, realistic deviations cannot account for such large discrepancy between the observed results and the predictions of the model of Ref. [2]. This suggests that either the model of Ref. [2] has to be ruled out or the parameter regime originally considered has to be revisited, e.g., by assuming much lower wormhole densities.

\section{CONCLUSION}

Ellis et al. have suggested more than 20 years ago that a hypothetical space-time configuration can degrade the coherence of spatial superposition states. We have shown that recently published data from atom interferometry rule out this model in the parameter regime originally proposed. The fact that the quantum-gravity-inspired model [2] can be ruled out by an interference experiment with ${ }^{87} \mathrm{Rb}$ atoms (rather light objects) might seem surprising at first sight. This can be understood, however, from the localization rate proportional to $\gamma_{\mathrm{QG}} d^{2}$ of the model of Ref. [2], which depends quadratically on the mass of the system $m$, but also on the spatial separation $d$ between the two arms of the interferometer. Remarkably, the $d^{2}$ dependence of the localization rate is not unique to the model of Ref. [2] and holds whenever the separation $d \ll \lambda$ is much smaller than the characteristic wavelength of the hypothetical field that induces the decoherence, be it wormholes, dark matter [43,44], or dark energy, such as chameleon fields [45-47]. This makes atom interferometry a very promising technique to probe these models. As future work, it might be interesting to see how the results of Ref. [38] bound other collapse models [48] in the spirit of what has been done in Refs. [49,50], for example. 


\section{ACKNOWLEDGMENTS}

J.M. would like to thank E. Copeland, C. Burrage, and A. Padilla for useful discussions. We thank M. Ho for a careful reading of the manuscript. This work was supported by Grant
No. EU-FET HAIRS 612862, the Swiss National Science Foundation Grant No. PP00P2-150579, and the Austrian Science Fund (Grants No. P24273-N16 and No. P28000-N27).
[1] J. Ellis, J. S. Hagelin, D. V. Nanopoulos, and M. Srednicki, Nucl. Phys. B 241, 381 (1984).

[2] J. Ellis, S. Mohanty, and D. V. Nanopoulos, Phys. Lett. B 221, 113 (1989).

[3] J. Ellis, N. E. Mavromatos, and D. V. Nanopoulos, Phys. Lett. B 293, 37 (1992).

[4] N. E. Mavromatos, Planck Scale Effects in Astrophysics and Cosmology (Springer, Berlin, 2005), p. 245.

[5] S. Hossenfelder, in Classical and Quantum Gravity: Theory, Analysis and Applications, edited by V. R. Frignanni (Nova, Hauppauge, 2011), Chap. 5.

[6] S. Coleman, Nucl. Phys. B 310, 643 (1988).

[7] W. Fischler and L. Susskind, Phys. Lett. B 217, 48 (1989).

[8] J. Preskill, Nucl. Phys. B 323, 141 (1989).

[9] S. Coleman and K. Lee, Phys. Lett. B 221, 242 (1989).

[10] J. Polchinski, Nucl. Phys. B 325, 619 (1989).

[11] T. Banks, L. Susskind, and M. E. Peskin, Nucl. Phys. B 244, 125 (1984).

[12] S. W. Hawking, Phys. Scr. T36, 222 (1991).

[13] A. Padilla, arXiv:1502.05296.

[14] J. Ellis, N. Mavromatos, and D. V. Nanopoulos, Phys. Lett. B 293, 142 (1992).

[15] A. K. Alok, S. Banerjee, and S. U. Sankar, Phys. Lett. B 749, 94 (2015).

[16] J. Ellis, S. Mohanty, and D. V. Nanopoulos, Phys. Lett. B 235, 305 (1990).

[17] T. Spiller, J. Ralph, T. Clark, R. Prance, and H. Prance, Phys. Lett. B 244, 333 (1990).

[18] I. C. Percival and W. T. Strunz, Proc. R. Soc. A 453, 431 (1997).

[19] F. Benatti and R. Floreanini, Phys. Rev. A 66, 043617 (2002).

[20] F. Benatti and R. Floreanini, J. Opt. B 4, S238 (2002).

[21] C. Simon and D. Jaksch, Phys. Rev. A 70, 052104 (2004).

[22] C. H. Wang, R. Bingham, and J. T. Mendonça, Class. Quantum Grav. 23, L59 (2006).

[23] C. J. Hogan, Phys. Rev. D 85, 064007 (2012).

[24] B. Pepper, E. Jeffrey, R. Ghobadi, C. Simon, and D. Bouwmeester, New J. Phys. 14, 115025 (2012).

[25] M. Paternostro, L. Mazzola, and J. Li, J. Phys. B 45, 154010 (2012).

[26] P. Sekatski, M. Aspelmeyer, and N. Sangouard, Phys. Rev. Lett. 112, 080502 (2014).

[27] R. Ghobadi, S. Kumar, B. Pepper, D. Bouwmeester, A. I. Lvovsky, and C. Simon, Phys. Rev. Lett. 112, 080503 (2014).

[28] M. Bahrami, M. Paternostro, A. Bassi, and H. Ulbricht, Phys. Rev. Lett. 112, 210404 (2014).
[29] G. Hechenblaikner, F. Hufgard, J. Burkhardt, N. Kiesel, U. Johann, M. Aspelmeyer, and R. Kaltenbaek, New J. Phys. 16, 013058 (2014).

[30] W. Ge and M. S. Zubairy, Phys. Rev. A 91, 013842 (2015).

[31] M. Ho, A. Lafont, N. Sangouard, and P. Sekatski, New J. Phys. 18, 033025 (2016).

[32] V. C. Vivoli, T. Barnea, C. Galland, and N. Sangouard, Phys. Rev. Lett. 116, 070405 (2016).

[33] O. Romero-Isart, Phys. Rev. A 84, 052121 (2011).

[34] S. Bera, B. Motwani, T. P. Singh, and H. Ulbricht, Sci. Rep. 5, 7664 (2015).

[35] S. Colin, T. Durt, and R. Willox, Phys. Rev. A 93, 062102 (2016).

[36] R. Kaltenbaek, M. Aspelmeyer, P. F. Barker, A. Bassi, J. Bateman, K. Bongs, S. Bose, C. Braxmaier, Č. Brukner, B. Christophe et al., EPJ Quantum Technol. 3, 1 (2016).

[37] J. Minář, P. Sekatski, R. Stevenson, and N. Sangouard, arXiv:1601.05381.

[38] T. Kovachy, P. Asenbaum, C. Overstreet, C. A. Donnelly, S. M. Dickerson, A. Sugarbaker, J. M. Hogan, and M. A. Kasevich, Nature (London) 528, 530 (2015).

[39] E. Joos and H. Zeh, Z. Phys. B 59, 223 (1985).

[40] M. R. Gallis and G. N. Fleming, Phys. Rev. A 42, 38 (1990).

[41] S.-w. Chiow, T. Kovachy, H.-C. Chien, and M. A. Kasevich, Phys. Rev. Lett. 107, 130403 (2011).

[42] The specific choice of the atomic motion corresponds to a smooth acceleration ramp such that the maximum distance $d_{\max }$ is reached after time $T$. Such motion is achieved (to a good approximation), e.g., in atomic interferometers with a large number of momentum transfer kicks (see Fig. 1b in [38]) or in atomic transport in optical lattices as we discuss in more detail in [37].

[43] C. J. Riedel, Phys. Rev. D 88, 116005 (2013).

[44] C. J. Riedel and I. Yavin, arXiv:1609.04145.

[45] C. Burrage, E. Copeland, and E. Hinds, J. Cosmol. Astropart. Phys. 2015, 042 (2015).

[46] P. Hamilton, M. Jaffe, P. Haslinger, Q. Simmons, H. Müller, and J. Khoury, Science 349, 849 (2015).

[47] H. Lemmel, P. Brax, A. Ivanov, T. Jenke, G. Pignol, M. Pitschmann, T. Potocar, M. Wellenzohn, M. Zawisky, and H. Abele, Phys. Lett. B 743, 310 (2015).

[48] A. Bassi, K. Lochan, S. Satin, T. P. Singh, and H. Ulbricht, Rev. Mod. Phys. 85, 471 (2013).

[49] A. Vinante, M. Bahrami, A. Bassi, O. Usenko, G. Wijts, and T. H. Oosterkamp, Phys. Rev. Lett. 116, 090402 (2016).

[50] M. Toroš and A. Bassi, arXiv:1601.03672. 